\title{
ARTICLE
}

Received 19 Jan 2016 | Accepted 11 Oct 2016 | Published 16 Nov 2016

DOI: $10.1038 /$ ncomms13509

OPEN

\section{Lack of Diaph3 relaxes the spindle checkpoint causing the loss of neural progenitors}

\author{
Devid Damiani ${ }^{1,2}$, André M. Goffinet ${ }^{1,2}$, Arthur Alberts ${ }^{3}$ \& Fadel Tissir ${ }^{1}$
}

The diaphanous homologue Diaph3 (aka mDia2) is a major regulator of actin cytoskeleton. Loss of Diaph3 has been constantly associated with cytokinesis failure ascribed to impaired accumulation of actin in the cleavage furrow. Here we report that Diaph3 is required before cell fission, to ensure the accurate segregation of chromosomes. Inactivation of the Diaph3 gene causes a massive loss of cortical progenitor cells, with subsequent depletion of intermediate progenitors and neurons, and results in microcephaly. In embryonic brain extracts, Diaph3 co-immunoprecipitates with BubR1, a key regulator of the spindle assembly checkpoint (SAC). Diaph3-deficient cortical progenitors have decreased levels of BubR1 and fail to properly activate the SAC. Hence, they bypass mitotic arrest and embark on anaphase in spite of incorrect chromosome segregation, generating aneuploidy. Our data identify Diaph3 as a major guard of cortical progenitors, unravel novel functions of Diaphanous formins and add insights into the pathobiology of microcephaly.

\footnotetext{
${ }^{1}$ Developmental Neurobiology Unit, Université catholique de Louvain, Institute of Neuroscience, Avenue Mounier 73, Box B1.73.16, Brussels 1200, Belgium. ${ }^{2}$ Developmental Neurobiology Unit, WELBIO, Institute of Neuroscience, Avenue Mounier B1.73.16, Brussels 1200, Belgium. ${ }^{3}$ Laboratory of Cell Structure and Signal Integration, Van Andel Research Institute, 333 Bostwick Avenue N.E., Grand Rapids, Michigan 49503, USA. Correspondence and requests for materials should be addressed to F.T. (email: fadel.tissir@uclouvain.be).
} 
$\mathrm{F}$ ormins constitute a family of 15 proteins in the mouse and human, characterized by the presence of two formin homology domains. By interacting with the growing ends of actin filaments, formins protect from capping, catalyse actin polymerization and regulate filament bundling into filopodia ${ }^{1-4}$, supporting the establishment and maintenance of cell polarity during development and in response to disease. Diaphanous formins, known in mammals as Diaph1, 2 and 3 (Diaph1-3) are a subgroup of the formin family related to Drosophila diaphanous ${ }^{5}$. Diaph1-3 exist in two forms. In the inactive 'locked' form, the carboxy-terminal diaphanous autoregulatory domain interacts with the upstream inhibitory domain. Activation occurs through binding of a small GTPase to the GTPase binding domain, which disrupts the interaction between diaphanous inhibitory domain and diaphanous autoregulatory domain, and releases the protein ends ${ }^{6}$.

In flies, mutations in the diaphanous gene generate defects in gametogenesis and neuroblast formation, with polyploidy attributed to compromised cytokinesis ${ }^{7}$. In mammals, Diaph1-3 mutations have been associated with local actin cytoskeleton dysfunctions. For instance, in Diaph1 and 2 double-knockout (Diaph1-2 dko) mice, whereas radial migration and layer formation of cortical excitatory neurons are largely unaffected, the tangential migration of cortical interneurons and neuroblasts from the adult neurogenic sub-ventricular zone (VZ) to the olfactory bulbs are impaired. F-actin organization is disrupted at the rear of migrating interneurons and neuroblasts, impairing the translocation of centrosome and nucleus ${ }^{8}$. Diaph3 accumulates in the cleavage furrow during transition from anaphase to telophase, and its depletion in dividing cells in vitro affects the amount of F-actin at the equatorial region ${ }^{4,9}$. In Diaph3-deficient mice, erythroid cells differentiate normally, but during the late divisions of erythroblasts, daughter cells fail to separate because of decreased accumulation of actin in the cleavage furrow ${ }^{10}$. Mice with overexpression of Diaph3 have anomalies in the inner, but not outer, hair cells. These mice exhibit hearing loss, suggesting an essential role for Diaph3 in assembly and/or maintenance of actin filaments in stereocilia ${ }^{11}$. In humans, a mutation in the region coding for $5^{\prime}$-untranslated region of the DIAPH3 messenger RNA results in two to threefold overexpression of the protein, leading to delayed onset, progressive deafness known as auditory neuropathy non-syndromic autosomal dominant 1 (ref. 12). Furthermore, a double hit in the DIAPH3 gene (a maternally inherited deletion on $13 \mathrm{q}$ and a point mutation in the paternal copy) was associated with autism ${ }^{13}$. In addition to its well-documented role in actin cytoskeleton, in vitro studies have implicated Diaph3 in the dynamics of microtubules (MTs). Diaph3 co-localizes with stable MTs and its overexpression is sufficient to generate and orient stable $\mathrm{MTs}^{14}$. Diaph3 can directly bind (and stabilize) MTs in an actin nucleationindependent manner ${ }^{15,16}$. Alternatively, by interacting with the MT tip proteins EB1 and adenomatous polyposis coli (APC), Diaph3 was proposed to serve as scaffold protein ${ }^{17}$.

A key feature of the mammalian cortex is the substantial growth of its germinal zones. At early stages of cortical development, neuroepithelial (NE) cells proliferate rapidly by symmetrical division, to amplify the pool of progenitors ${ }^{18}$. A tight regulation of the cell division machinery is therefore required, to ensure a correct mitotic process and even segregation of chromosomes between daughter cells. Although intensive research in cortical development and evolution has identified numerous genes that influence cortical progenitor cell division, much effort is still needed to fully understand the underlying molecular mechanisms. Here we report that the formin Diaph3 acts early in mitosis to secure appropriate karyokinesis. Diaph3 belongs to a molecular network that comprises components of the spindle assembly checkpoint (SAC) and chromosomal passenger complex (CPC) machineries. This network regulates kinetochores-mitotic spindle interactions and controls the transition of cortical progenitors from metaphase to anaphase. Mutation of Diaph3 compromises the level of SAC activation. Hence, nuclear errors are not properly 'amended' by the spindle checkpoint, causing aneuploidy, cell death and cortical hypoplasia.

\section{Results}

Diaph3 ko mice display severe developmental defects. We studied the expression of Diaph3 in the nervous system using in situ hybridization. The mRNA signal was diffuse at embryonic (E) day 10.5 and more confined to $\mathrm{VZ}$ of the cerebral cortex at E13.5 (Fig. 1a). Analysis using a fluorescent RNAscope probe showed that the signal was the highest in the outermost germinal zone, where radial glial and intermediate progenitor cells reside, and no signal was observed in doublecortin-positive neurons (Fig. 1b-e). To inactivate the Diaph3 gene, we inserted a cassette containing FRT-En2-IRES-LacZ-loxP-neo-FRT-loxP in intron 9 (Supplementary Fig. 1a). Appropriately targeted embryonic stem cells were cloned and injected in C57BL/6N blastocysts. To validate the mutation, we used complementary DNA from wild-type (WT), heterozygous $(k o /+)$ and $k o$ mice as a template for reverse transcriptase-PCR (Supplementary Fig. 1b). A PCR amplifying exons 8-9 was used as a control (present in the cDNAs from the three genotypes). Two primer pairs spanning exons 9-11 and 13-15 were predicted to produce no amplicon in ko mice. The two pairs produced however amplicons even in the $k o$. Primers in exons 9 and 11 yielded a larger amplicon than in WT. Sequencing of this amplicon showed the presence of a cryptic splicing donor site located 115 nucleotides downstream of the beginning of exon En2. The mRNA was spliced to exon 10, as in WT mice, but included 115 nucleotides from the cassette, creating a frameshift mutation with 3 stop codons in exon 10 (Supplementary Fig. 1c). This suggests that no functional Diaph3 protein could be produced in the $k o$ allele. To verify this, we analysed protein extracts from E13.5 embryos of WT, heterozygous and ko mice by western blotting using a rabbit polyclonal antibody against the C-terminal segment of the Diaph3 protein (Fig. 1f). The antibody disclosed a doublet in the $130 \mathrm{kDa}$ range in WT and heterozygous, but not in ko tissue (predicted Diaph3 molecular weight: $134 \mathrm{kDa}$ ), confirming the absence of the C-terminal fragment of the protein. Diaph $3^{k o /+}$ heterozygous mice were viable and fertile. Very few Diaph3 ko mice were born (5 instead of $\sim 200$ expected in 803 genotyped pups), indicating that the homozygous mutation is mostly embryonic lethal. Surviving animals displayed small brain and body size, as well as facial deformities (Fig. 1g-i). In addition, hydrocephalus was found in three out of the five mutants (Supplementary Fig. 2a).

As most of the Diaph3 mutants died before birth, we compared $k o$ and control (WT or heterozygous) embryos at different developmental stages. They looked quite similar up to E10.5 (Fig. 2a). From E11.5 onwards, ko embryos consistently exhibited developmental abnormalities, with small size, bending of the body axis and a drastic atrophy of the lower jaw (Fig. 2b). Mutant embryos with the most severe phenotype were also characterized by an axial tortuosity of the neural tube, ranging from a moderate bending to a clear zig-zag pattern (Fig. 2c). Based on the expression profile and brain phenotype, we investigated the development of the cerebral cortex. At E13.5, there was a consistent reduction of the size of dorsal telencephalon (Fig. 2d). The phenotype varied from mild to severe expressivity, yet occurred with a $100 \%$ penetrance. Moreover, an increase 
a
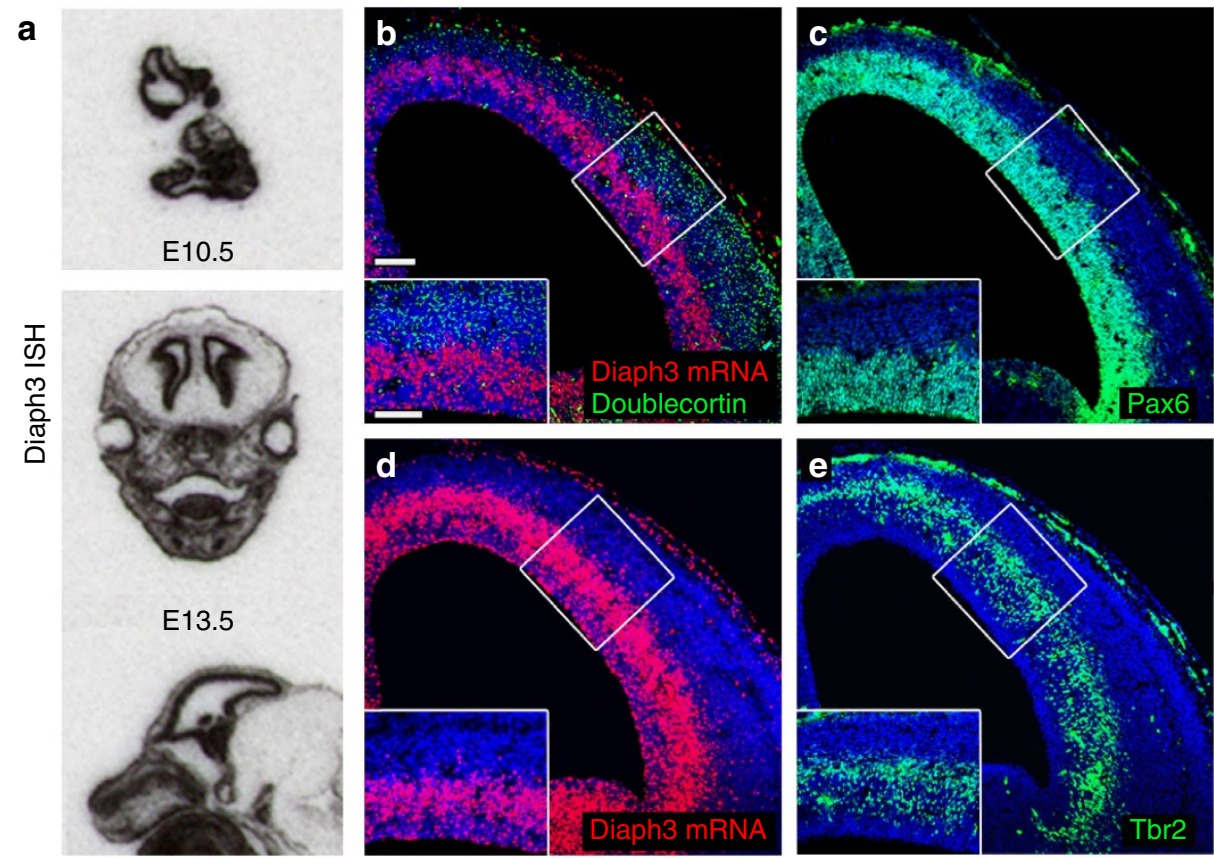

f
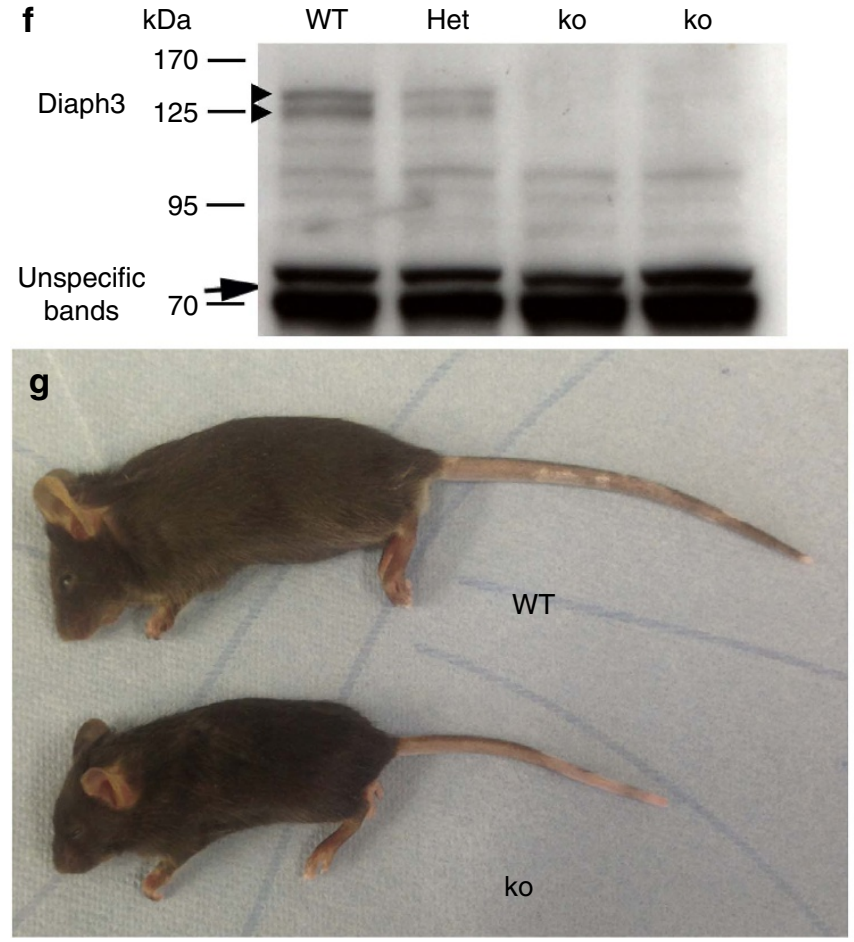

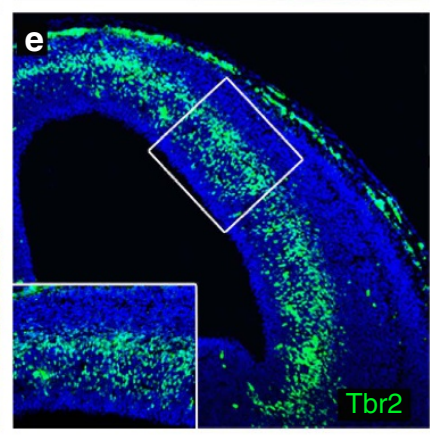

$\mathbf{h}$


Figure 1 | Generation of Diaph3 mutant mice. (a) Sections of E10.5 embryos and E13.5 heads, hybridized with a Diaph3 radiolabelled probe. The mRNA signal is associated with the telencephalic VZ where the progenitor cells reside. (b-e) Coronal sections of E13.5 brain hemispheres hybridized with a fast red-labelled RNAscope probe. The signal localized mostly in the outer part of the VZ (b,d). No signal was found in committed cortical neurons stained for Doublecortin (green, b). (c,e) Sections adjacent to those illustrated in $\mathbf{b}, \mathbf{d}$, stained for Pax6 (c) and Tbr2 (e). Scale bars, $100 \mu \mathrm{m}$ and $50 \mu \mathrm{m}$ in the insets. (f) Western blotting of E13.5 embryo lysates from WT, heterozygotes (Het) and mutant ( $k o$ ) littermates. The absence of Diaph3 protein doublet in Diaph3 ko mice (arrowheads) is noteworthy. Nonspecific bands at $70 \mathrm{kDa}$ provide a loading control (arrow). (g-i) Comparison between Diaph3 WT and ko littermates at P25. The small body size (g), small brains (h) and facial deformities (i) in Diaph3 ko mice are noteworthy. ISH, in situ hybridization.

in both the number and size of blood vessels was observed in haematoxylin-eosin- and Isolectin B4-stained sections (Fig. 2e and Supplementary Fig. 2b). Diaph3 ko embryos appeared whitish (Fig. 2f) and histological examination disclosed a substantial depletion of erythrocyte progenitors in hepatic erythroblastic islands that resulted in severe anaemia, confirming previous findings using a different mutant allele ${ }^{10}$.
The large majority ( 97.5\%) of mutant embryos died between E12.5 and E14.5.

Cortical hypoplasia is due to apoptosis of neural progenitors. We analysed the cytoarchitecture of cerebral cortex by immunohistochemistry. At E13.5, the number of the three main 
a

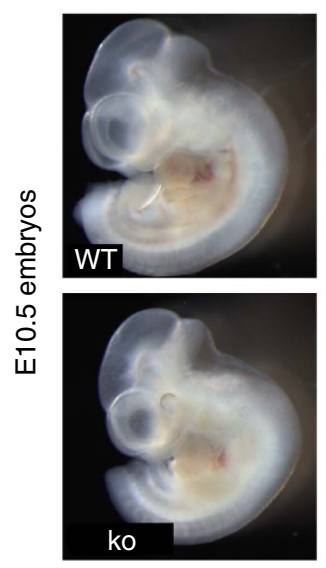

d

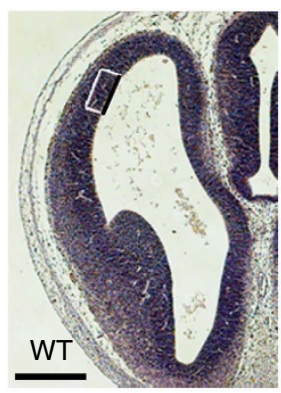

e

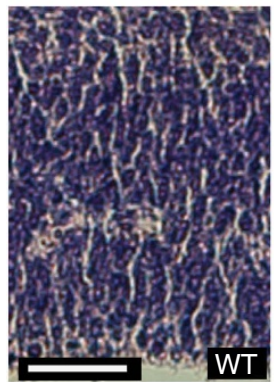

b
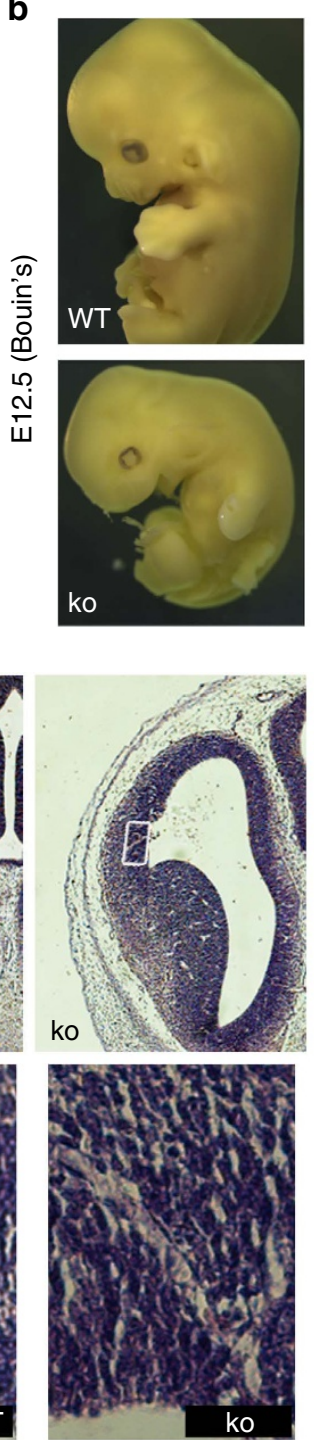

C



$\mathbf{f}$

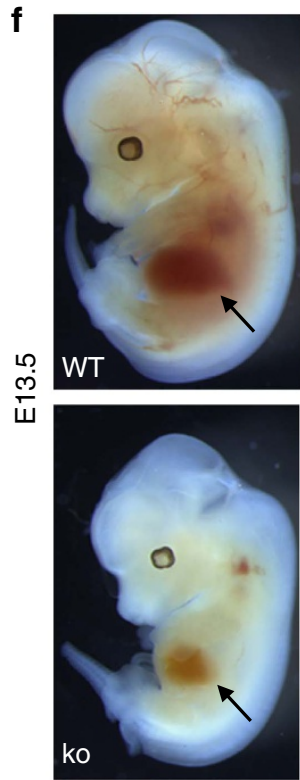

Figure 2 | Developmental phenotype of Diaph3 mutant mice. (a) Development of Diaph3 mutant mice was grossly normal until E10.5. (b) At E12.5, mutant embryos displayed a delayed development and facial malformation. (c) Neural tube deformity in Diaph3 mutant embryos. A bending pattern was seen in severely affected mutants. (d) Haematoxylin-eosin-stained sections of E13.5 telencephalon from WT and Diaph3 ko littermates. The reduction of cortical size was already evident at this stage. (e) High magnification of the boxed areas in d, showing the enlargement of blood vessels in the mutant embryos. (f) Whitish appearance and impaired liver haematopoiesis in E13.5 ko compared with WT embryos. Scale bars, $500 \mu \mathrm{m}$ (d) and $50 \mu \mathrm{m}$ (e).

cell populations, namely apical (radial glial) progenitors $\left(\mathrm{Pax}^{+}\right)$, intermediate progenitors $\left(\mathrm{Tbr} 2^{+}\right)$and neurons $\left(\mathrm{Tbr} 1^{+}\right)$. The three populations were decreased in Diaph3 ko mice (Fig. 3a,b; quantification in Fig. 3c). Compared with WT littermates, the number of apical progenitors declined by $\sim 40 \%$ (number of $\mathrm{Pax}^{+}$cells per $100 \mu \mathrm{m}$-wide stripe of frontal cortex: $243.75 \pm 15.75$ versus $377.40 \pm 22$; medial cortex: $188.6 \pm 7.61$ versus $299.44 \pm 22.39$; caudal cortex: $159.4 \pm 4.81$ versus $233.7 \pm 20.31)$. A comparable reduction was observed in the number of intermediate progenitors (number of Tbr ${ }^{+}$cells per $100 \mu \mathrm{m}$-wide stripe of frontal cortex: $140.32 \pm 10.6$ versus $238.66 \pm 15.06$; medial cortex: $103.74 \pm 5.04$ versus $182.3 \pm 12.56$; caudal cortex $103.84 \pm 8.11$ versus $145.05 \pm 9.83$ ) and postmitotic neurons (number of $\mathrm{Tbr}^{+}$cells per $100 \mu \mathrm{m}$-wide stripe of frontal cortex: $100.78 \pm 5.32$ versus $157.24 \pm 11.56$; medial cortex: $71.95 \pm 4.35$ versus $125.7 \pm 12.42$; caudal cortex: $59.05 \pm 6.34$ versus $138.81 \pm 11.53)$. Thus, the three cortical cell types were reduced in E13.5 Diaph3 ko embryos. To understand the developmental basis of cortical hypoplasia, we studied apoptosis using immunohistochemistry for activated Caspase-3aCas and TdT-mediated dUTP nick end labelling (TUNEL). We observed several aCas3-positive cells in the mutant cortex at E13.5, whereas such cells were hardly seen in control littermates (Fig. 3d). We examined earlier stages and detected a massive cell death at E10.5 (Fig. 4a,b). Many cells were also positive for aCas9 (Fig. 4c), suggesting that mutant cells die via the mitochondrial apoptosis pathway ${ }^{19-21}$. Double immunostaining indicated that the dying cells $\left(\mathrm{aCas}^{+}\right.$or $\mathrm{TUNEL}^{+}$) were neither neurons $\left(\right.$Tuj $1^{+}$) nor intermediate progenitors $\left(\mathrm{Tbr} 2^{+}\right)$, but rather $\mathrm{Pax}^{+}$neural progenitors (Fig. 4d-g and Supplementary Fig. 3a,b).

Given the defective erythropoiesis in Diaph3 mutant mice, we wondered whether the reduced erythrocyte concentration could trigger hypoxia and induce apoptosis of cortical progenitor cells. In E10.5 embryos, blood vessels have not yet invaded the neuroepithelium and remain confined to meninges. Brain tissue oxygenation depends on local diffusion rather than intra-parenchymal circulation. Nevertheless, the possibility that the oxygen pressure is perturbed in Diaph 3 ko embryos cannot be 

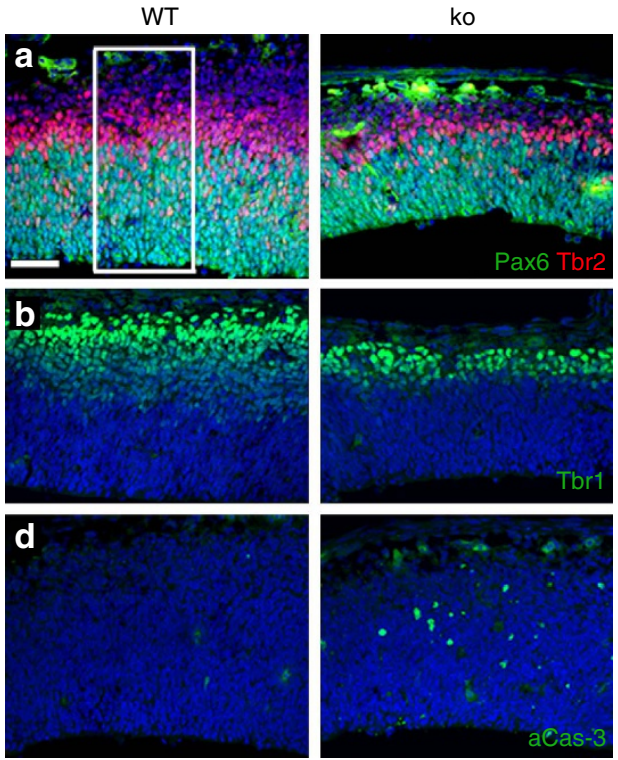

C

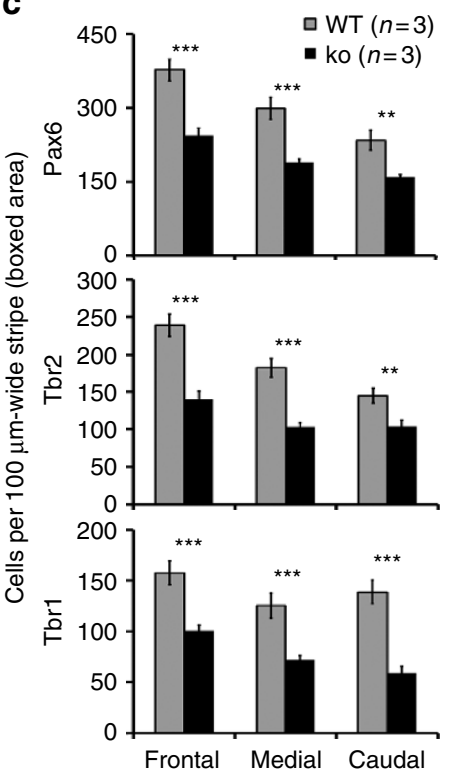

Figure 3 | Different cell populations are affected in Diaph3 mutant. E13.5 cortical sections immunostained for Tbr2 and Pax6 (a), Tbr1 (b) and aCas3 (d). (c) Quantification of Pax6 ${ }^{+}, \mathrm{Tbr}^{+}$and Tbr1 ${ }^{+}$cells. Cells were counted in $317.13 \mu \mathrm{m}$-wide cortical areas from three mutants and three controls, and counts were expressed as cells per $100 \mu \mathrm{m}$-wide cortical stripe (boxed area in a). An average of $40 \%$ decrease in the three cell populations was seen in the mutant tissue. $n=3$ ko and 3 WT embryos, Student's $t$-test, ${ }^{\star \star} P<0.01$ and ${ }^{\star \star \star} P<0.001$. Error bars represent s.e.m. Scale bar, $50 \mu \mathrm{m}$.

completely excluded. To test this, we injected pregnant females at E10.5, when cortical cell death peaks, with the hypoxyprobe-1 (pimonidazole hydrochloride), which binds covalently to proteins when the oxygen pressure is lower than $10 \mathrm{~mm} \mathrm{Hg}$. As a positive control, we used hypoxic tumours obtained by subcutaneous injection of human breast cancer-derived cells (B16F10) in mice. The staining with anti-pimonidazole antibodies was very low and comparable in $k o$ and control cortices, whereas intense staining was visible in the tumour tissue (Supplementary Fig. 4). To definitely rule out a role of hypoxia-induced apoptosis, we generated cortex-specific mutants by crossing the Diaph3 floxed allele (exons 10 and 11 are flanked by LoxP sites) with Emx1-Cre mice $^{22}$ (where the Cre recombinase is active in cortical cells from E9; Supplementary Fig. 1a). Whereas no apoptosis was seen in Diaph $3^{f /+}$;Emx1-Cre or Diaph3 $3^{f / f}$ cortices, Diaph3 $3^{f / f}$;Emx1-Cre embryos exhibited many apoptotic cells, demonstrating that cell death is caused by the lack of Diaph3 in cortical progenitors rather than in blood cells (Supplementary Fig. 5a). The loss of cortical progenitors led to a significant reduction of cortical size at E15.5 and in the adult (Supplementary Fig. 5b-d).

Mitotic errors generate aneuploidy and cell death. As many apoptotic profiles scattered throughout the cortex resembled cell remnants with a round morphology and two or more micronuclei sporadically (Fig. 4b), we focused on mitotic cells located in the apical tier of the VZ. 4,6-Diamidino-2-phenylindole (DAPI) staining indicated the presence of asymmetric nuclear division (Fig. 4g). We examined 211 dividing (phospho-Vimentin ${ }^{+}$) cells, among which 71 were apoptotic $\left(\mathrm{aCas}^{+}\right.$or $\left.\mathrm{TUNEL}^{+}\right)$. Whereas only $6.4 \%(9 / 140)$ of non-apoptotic cells displayed mitotic errors, 98.5\% (70/71) of apoptotic cells had such errors, strongly suggesting a correlation between apoptosis and chromosome segregation abnormalities in Diaph 3 ko cortical progenitor cells (Fig. 4h and Supplementary Fig. 3c,d). These abnormalities produce mitotic catastrophe or give rise to aneuploid progenies ${ }^{23}$. To assess whether the dying cells, scattered in the Diaph3 ko dorsal telencephalon, were aneuploid offspring of aberrantly dividing cortical progenitors, we quantified the genomic content of E11.5 telencephalic cells by DNA flow cytometry analysis. We found that, in addition to the $2 \mathrm{C}$ and $4 \mathrm{C}$ peak characteristics of euploid cells before and after DNA replication, respectively, a significant fraction of mutant cells exhibited a DNA content less than $2 \mathrm{C}(k o: 5.43 \pm 1.20 \%, n=21,233$ cells from 5 forebrains, control: $0.73 \pm 1.20 \%, n=125,123$ cells from 23 forebrains; Fig. 4i). Both asymmetric segregation and abnormal genomic content in the mutant tissue prompted us to analyse the karyotype of Diaph3-deficient neural stem cells, grown as neurospheres. We confirmed the neural identity by Nestin immunostaining (Supplementary Fig. 6). We counted the number of chromosomes in 129 control and 161 mutant metaphase spreads obtained from cells harvested at passage 8 (4 animals for each genotype). In control cells, $90 \%$ of metaphases $(116 / 129)$ were euploid and the number of chromosomes in aneuploid metaphases comprised between 38 and 42 (Fig. $4 j$ and Supplementary Table 1). In sharp contrast, the percentage of euploid metaphases decreased to $35 \%$ (57/161) in mutant cells. Among aneuploid cells, $16.1 \%(26 / 161)$ had $<38$ chromosomes (Fig. 4k), 6.2\% (10/161) had >42 chromosomes (Fig. 4l) and $3.7 \%(6 / 161)$ were tetraploid (Fig. 4m). Taken together, these results suggest that the lack of Diaph3 disrupts nuclear segregation and triggers chromosomal instability, aneuploidy and loss of cortical progenitors.

Absence of Diaph3 relaxes the spindle checkpoint. During mitosis, segregation of chromosomes relies on the SAC machinery, which 'monitors' connections between spindle MTs and kinetochores, and delays the transition to anaphase until all chromosomes are correctly attached. Given the chromosomal instability in Diaph3 ko mice, we anticipated an overactivation of the SAC, with accumulation of dividing neural progenitors and overall increase in mitotic cell density. We tested this hypothesis using immunohistochemistry for phosphorylated histone $\mathrm{H} 3$ ( $\mathrm{pHH} 3$, mitotic cells) and BubR1, a protein that accumulates at unattached kinetochores and diffuses to the cytoplasm, to inhibit 

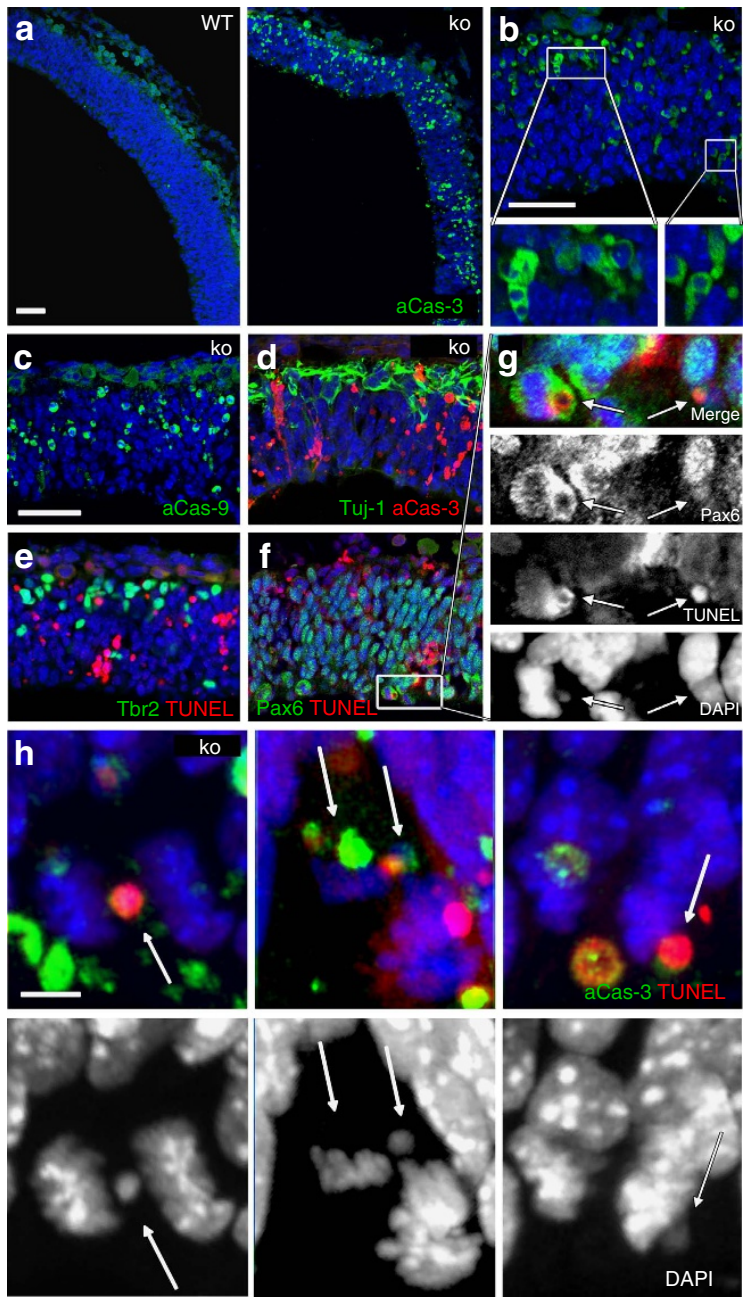

i
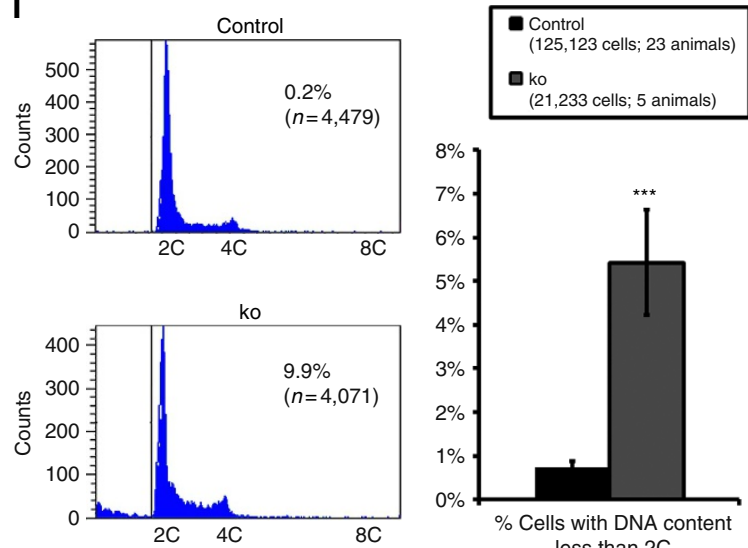

less than $2 \mathrm{C}$
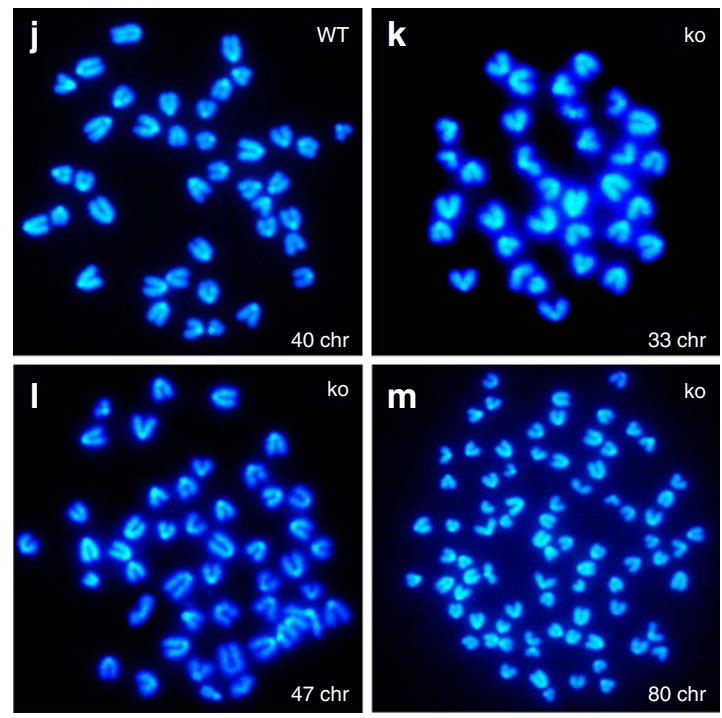

Figure 4 | Cell death and aneuploidy in Diaph3 ko mice. (a-h) E10.5 Cortical sections stained for aCas3 (a,b), aCas9 (c), aCas3 and Tuj1 (d), Tbr2 and TUNEL (e), Pax6 and TUNEL (f,g), and aCas3 and TUNEL (h). Widespread apoptotic profiles were found in the mutant (a,b). Immunoreactivity for aCas 9 points to the mitochondrial apoptotic pathway (c). Co-staining with Tuj1 (neurons, d), Tbr2 (intermediate progenitors, e) and Pax6 (radial glial progenitor cells, f) identified the latter as the dying population. Apoptotic cells (TUNEL and/or aCas3, $\mathbf{g}, \mathbf{h}$ ) in the VZ showed asymmetric nuclear segregation (g), with in some cases the presence of aberrant nuclear profiles and micronuclei (arrows in $\mathbf{g}, \mathbf{h}$ ). Scale bars, $50 \mu \mathrm{m}$ (a-f) and $5 \mu \mathrm{m}$ (h). (i) Abnormal genomic content in mutant cortical progenitors. The DNA content is plotted in $x$ axis and the number of cells (Count) in the $y$ axis. The two main peaks represent DNA content of cells before (2C) and after (4C) S phase. A significant fraction of mutant cells had a DNA content less than $2 \mathrm{C}(5.43 \pm 1.2 \%$ of mutant cells versus $0.73 \pm 0.12 \%$ of control cells; $n=125,123$ control cells from 23 animals and 21,233 ko cells from 5 animals, $P<0.0001 ; z$-test). Error bars represent s.e.m. (j-m) Metaphase spreads prepared from WT (j) and mutant $(\mathbf{k}-\mathbf{m})$ neurospheres. Metaphases with less $(\mathbf{k})$ and more $(\mathbf{I})$ than forty chromosomes (chr) were frequent in mutant clones. Tetraploid metaphases were also occasionally observed (m).

progression to anaphase ${ }^{24}$ (Fig. 5a-d). Unexpectedly, the percentage of $\mathrm{BubR} 1^{+} / \mathrm{pHH}^{+}$cells in pre-anaphase (that is, dividing cells that activate the SAC and arrest in metaphase) was decreased in the mutant tissue (WT: $65.05 \pm 1.62 \%, k o$ : $53.26 \pm 0.78 \%, n=1,473$ cells from $3 \mathrm{WT}$ animals and 1,144 cells from 3 ko animals; $P<0.0001$; Fig. 5e). Furthermore, the overall density of mitotic cells declined considerably (WT: $8.38 \pm 0.29$ cells per $100 \mu \mathrm{m}$ of VZ, $k o: 6.22 \pm 0.23$ cells per $100 \mu \mathrm{m}$ of VZ, $P<0.0001$; Fig. 5f), whereas the fraction of postmetaphasic cells among mitotic cells increased in Diaph 3 ko tissue (WT: $27,8 \pm 2.06 \%, k o: 39.91 \pm 3.2 \%, n=786$ cells from 6 WT animals and 889 cells from $5 \mathrm{ko}$ animals; Fig. 5g). These results indicate that mutant cortical progenitor cells are less sensitive to nuclear division errors and do not systematically halt in metaphase, in spite of chromosome mis-segregation. They also suggest that the stringency of the SAC is downregulated in Diaph 3 ko. Given that haploinsufficiency of BubR1 is sufficient to cause aneuploidy in mice and humans ${ }^{25,26}$, and that Diaph3 interactors APC and EB1 regulate the alignment and segregation of chromosomes through interaction with BubR1 (refs 27,28), we assessed the amount of the SAC protein BubR1. Western blot analysis of telencephalon extracts showed an $\sim 50 \%$ reduction of BubR1 levels in mutant brain tissue (Fig. 5h,i). Moreover, BubR1 could be immunoprecipitated from cortical lysates of Diaph3 transgenic (TG) mice using anti-Diaph3 antibodies (Fig. 5j). To further explore the link between Diaph3 and BubR1, we infected Diaph3-deficient cortical progenitors with lentiviruses expressing either green fluorescent protein (GFP) or BubR1-IRES-GFP, cultured them as neurospheres for four passages and analysed their karyotype. Whereas the number of aneuploid metaphases was $60.5 \%$ in GFP-infected neurospheres, this percentage dropped to $33.9 \%$ in the BubR1-IRES-GFPinfected neurospheres (Supplementary Fig. 7), indicating that BubR1 overexpression reduced the number of aneuploid cells and rescued partially the Diaph3 phenotype. Conversely, we downregulated BubR1 by small interfering RNAs (siRNAs) in 
a



b

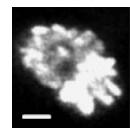

$\mathrm{pHH} 3$

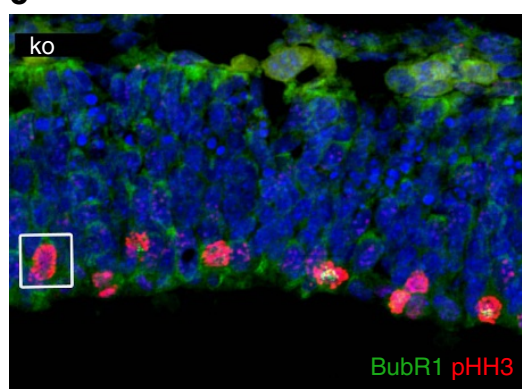

d

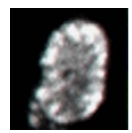

$\mathrm{pHH} 3$

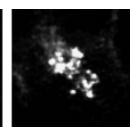

BubR1

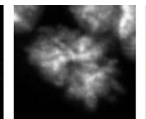

DAPI

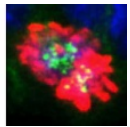

Merge

\section{e}

$\mathrm{BubR}^{+} / \mathrm{pHH}^{+}$(pre-anaphase)

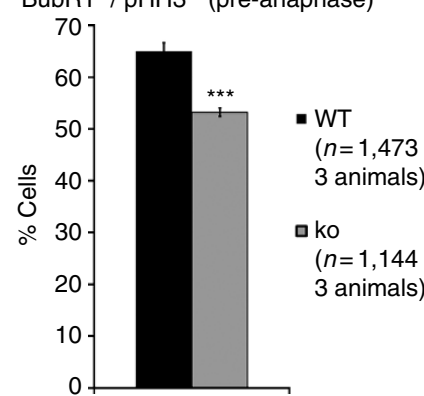

f

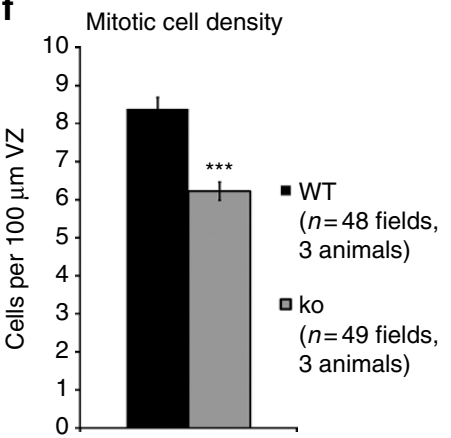

g



h

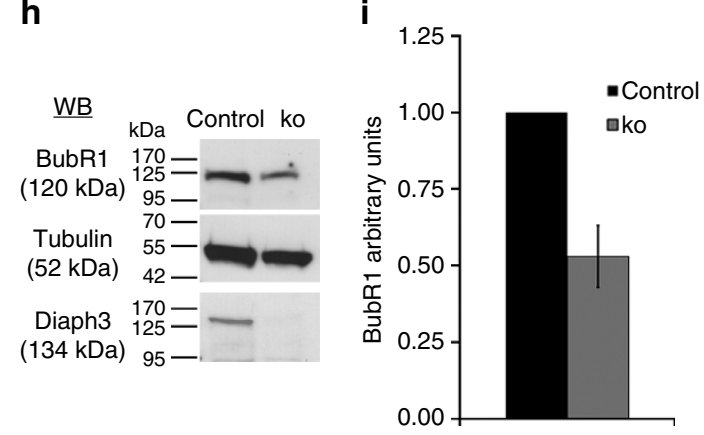

j
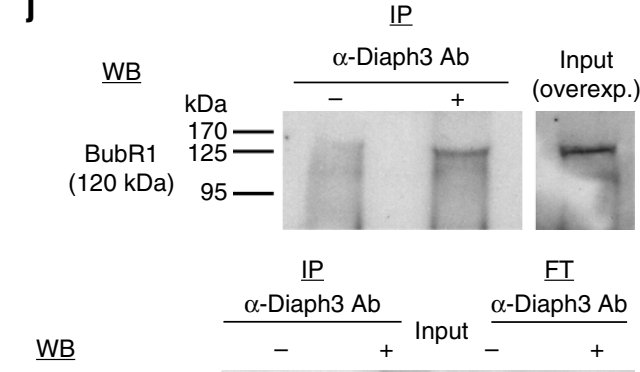

$\underline{W B}$

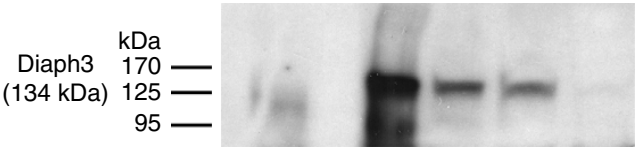

Figure 5 | Lack of Diaph3 disables the SAC. (a-d) Immunostaining for BubR1 (green) and pHH3 (red) of cortical sections from WT (a) and Diaph3 ko (c) E10.5 embryos. (b,d) Enlargements of the boxed areas in a,c, respectively. BubR1 (a hallmark of SAC activation) accumulated more in normal than in Diaph3-deficient cells (quantification in $\mathbf{e} ; n=1,473$ control cells from 3 animals and 1,144 ko cells from 3 animals; $P<0.0001, z$-test). (f) The density of mitotic cells in the cortex of E10.5 embryos was lower in the ko than in WT ( $n=48$ WT and 49 ko of $100 \mu$ m wide cortical stripes from 3 animals each genotype; $P<0.0001$; Student's t-test). (g) Quantification of postmetaphasic cells in the population of mitotic cells. The percentage of mitotic cells that underwent the metaphase-anaphase transition was higher in the ko than in control littermates ( $n=786$ control cells from 6 animals and 889 ko cells from 5 animals; $P<0.0001, z$-test). (h,i) Reduction of BubR1 protein levels in the mutant telencephalon detected by western blotting and quantified relatively to tubulin. The same membranes were blotted for Diaph3 to confirm the absence of the protein (h). The level of BubR1 decreased by half in the ko (i; $n=15$ embryos in 4 pools for each genotype). (j) Western blotting (WB) detects BubR1 on IP with anti-Diaph3 antibodies from TG cortical lysates. Diaph3 was used as a positive control for the IP (Input). No signal was found in the eluted fraction (flow-through, FT) in presence of Diaph3 antibodies. Detection of the protein in the lysate (Input) required overexposure of the film. Scale bars, $50 \mu \mathrm{m}(\mathbf{a}, \mathbf{c})$ and $5 \mu \mathrm{m}(\mathbf{b}, \mathbf{d})$. Error bars represent s.e.m.

WT neurospheres and did not detect any change in Diaph3 levels by western blotting (Supplementary Fig. 8a). Knockdown of BubR1 in mouse embryonic fibroblasts (MEFs) derived from TG embryos overexpressing Diaph3 and control littermates led to a substantial loss of MEFs in both genotypes. In addition, the percentage of aneuploid metaphases was $42 \%$ in control MEFs and $44.6 \%$ in Diaph3 TG MEFs, suggesting that the overexpression of Diaph3 was not sufficient to prevent aneuploidy and cell death induced by BubR1 downregulation (Supplementary Fig. 8b,c). We used siRNA to knock down the human DIAPH3 in in the hypotriploid HEK293T cells ${ }^{29}$ (Supplementary Fig. 9). Karyotype analysis showed that in cells treated with control siRNAs, $65 \%$ of metaphases had a chromosome number ranging from 50 to $84,15 \%$ had between 21 and 49 chromosomes, and $19 \%$ had $<20$ chromosomes. In DIAPH3-siRNA-treated cells, $27 \%$ of metaphase spreads had between 50 and 70 chromosomes, 19\% had between 21 and 49 chromosomes, and $49 \%$ had $<20$ chromosomes. Remarkably, DIAPH3 downregulation resulted in a concomitant reduction of BUBR1 levels, suggesting that, similar to that in mice, the downregulation of DIAPH3 in human cells disturbs karyokinesis and promotes aneuploidy. Collectively, these results establish a relationship between Diaph3, BubR1 and chromosome segregation during division of cortical progenitors. 
Key to the SAC activity is the $\mathrm{CPC}$, formed by Aurora kinase B, Survivin, Borealin and Incenp. CPC is located on centromeric DNA and 'senses' the tension between sister chromatids at the kinetochore ${ }^{30}$. In case of improper connections, Aurora B phosphorylates kinetochore proteins, thereby destabilizing spindle MT-kinetochore interactions ${ }^{31,32}$. To explore the relationship between Diaph3 and CPC in cortical progenitors in vivo, we examined the subcellular localization of the Diaph3 protein on in utero electroporation of a plasmid coding for a GFP::Diaph3 fusion protein ${ }^{3}$ in E13.5 embryos. One day after electroporation, the GFP was present in dorsal telencephalic cells. The signal was perinuclear and extended to apical and basal processes in non-dividing cells (Supplementary Fig. 10, arrowheads) ${ }^{33}$ and more diffusely distributed around the chromatin in dividing cells (Supplementary Fig. 10, arrows) ${ }^{34,35}$. We carried out co-immunoprecipitation (IP) experiments on E13.5 brain extracts from Diaph3 TG mouse ${ }^{11}$ and detected a previously uncharacterized interaction between Diaph3 and Survivin (Fig. 6a). Thus, Diaph3 interacts with CPC components during division of cortical progenitor cells. As Diaph3 was shown to form a complex with APC and EB1 at the plus-end of $\mathrm{MT}^{17}$, and APC and EB1 control the centromeric localization ${ }^{36}$ and stability $^{37}$ of the CPC component Aurora B, we studied the

a

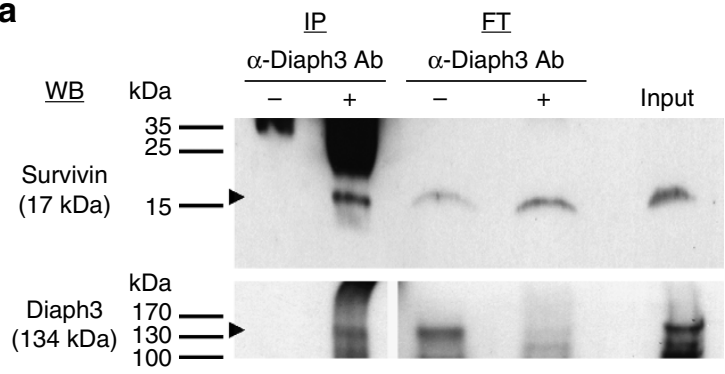

b
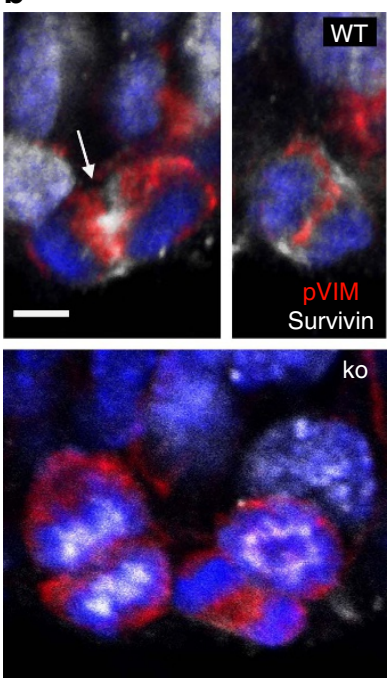

C

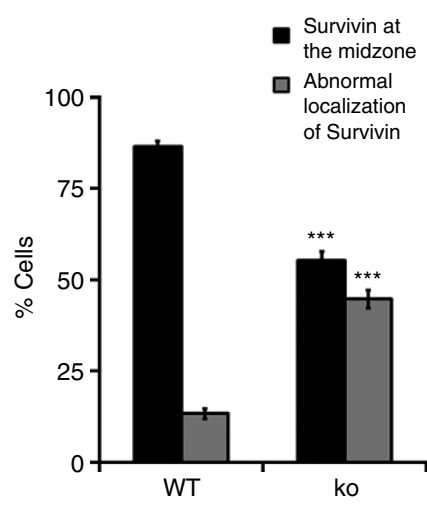

Figure 6 | Diaph3 interacts with Survivin. (a) Co-immunoprecipitation of endogenous Survivin and Diaph3 from brain lysates of Diaph3 TG mice. Western blotting was positive for Survivin on anti-Diaph3 immunoprecipitation $(+)$. No signal was present in absence of antibodies $(-)$. Western blotting for Diaph3 was used as a positive control. FT, flow-through. (b) Unlike in WT postmetaphasic cells, where Survivin is located at the midzone (arrow), this localization was disturbed in the Diaph3 ko. (c) Quantification of postmetaphasic cells with normal or abnormal distribution of Survivin ( $n=193$ control cells and 288 ko cells; $P<0.0001$, $z$-test). Error bars in c represent s.e.m. Scale bar, $10 \mu \mathrm{m}$. localization of CPC proteins in mutant and control neural progenitors. In dividing WT progenitors, CPC proteins relocate to the midzone after chromosome separation, to prevent SAC activation due to loss of tension ${ }^{38}$. In agreement, we found that CPC protein Survivin localized at the midzone in most dividing cells beyond the stage of metaphase ( $86.61 \pm 1.40 \%, n=193$ cells). By contrast, a substantial fraction $(44.68 \pm 2.41 \%, n=288$ cells) of post-metaphasic mutant cells displayed an atypical distribution of Survivin (Fig. 6b,c).

\section{Discussion}

In this work, we generated and analysed a mouse line carrying a mutation in the Diaph3 gene. In the ko mice, cortical progenitor cells undergo apoptosis as early as E10.5. Using flow cytometry analysis, we found a sevenfold increase in the proportion of aneuploid cells in the mutant telencephalon $(5.43 \%$ versus $0.73 \%)$. These cells presumably die, depleting progressively the population of progenitors and leading to cortical hypoplasia, as shown by the marked reduction in all cortical cell types in Diaph3 ko embryos at E13.5. Aneuploidy could eventually give rise to neoplastic transformation. Remarkably, mutations in the human $D I A P H 3$ gene are frequently found in metastatic cancers and downregulation of DIAPH3 increases metastatic invasion in xenotransplanted mice ${ }^{39,40}$. The nuclear asymmetric division we report here could increase chromosomal instability, promoting the emergence of new mutations and facilitating the acquisition of metastatic properties.

Karyotype analysis of neurospheres derived from Diaph 3 ko NE cells revealed that $65 \%$ of mutant cells had chromosome numerical abnormalities. The high prevalence of aneuploidy in neurosphere cultures was unexpected in view of the flow cytometry results (65\% in vitro versus $5 \%$ in vivo). However, several factors can account for this apparent discrepancy. First, cells with 38, 39, 41 or 42 chromosomes, which are the most abundant among the aneuploid cell population, are not easily distinguishable by flow cytometry, as their DNA content is close to that of euploid cells. Second, aneuploid cells with a DNA content higher than $2 \mathrm{C}$ cannot be discriminated from euploid cells in $\mathrm{S}$ phase, which also have a DNA content between $2 \mathrm{C}$ and $4 \mathrm{C}$, and were not taken into account in the flow cytometry analysis. Third, contrary to the developing brain, where aneuploid cells are mostly eliminated by apoptosis, such cells accumulate in vitro, probably because culture conditions promote their viability. Interestingly, the incidence of aneuploidy in Diaph3-deficient neurospheres is comparable to that of cultured MEFs carrying a hypomorphic mutation of BubR1 (ref. 41). Even though tetraploidy was associated with other chromosome numerical abnormalities as in the mosaic variegated aneuploidy ${ }^{26}$, we cannot exclude the possibility that tetraploid cells arose from cytokinesis failure. Diaph3 has been associated with actin dynamics in vitro ${ }^{4,9,10}$. In vivo, the lack of Diaph3 disrupts the accumulation of filamentous actin in the contractile ring, impairing cytokinesis of erythroid cells and generating multinucleated cells. We did not observe overt modifications of actin cytoskeleton in neuropithelial cells in Diaph3 ko at early embryonic stages, suggesting that depletion of Diaph3 may not affect all cells similarly. The fact that early erythroid precursors are not affected by the mutation ${ }^{10}$ supports this assumption. In those cells, similar to that in cortical progenitors, Diaph proteins are coexpressed and could have redundant functions in the formation of the contractile ring.

Diaph3 co-immunoprecipitates with the mitotic spindle protein BubR1 and its mutation reduces by half the overall level of BubR1. We did not see any accumulation of cells in metaphase. Thus, the lack of Diaph3 weakens the spindle checkpoint and behaves as a BubR1 hypomorphic (h) allele. In support of this, the phenotype of Diaph 3 ko phenocopies that of $B u b R 1^{h}$ mice in 
which mitotic slippage, formation of micronuclei, premature chromatid separation, aneuploidy and decreased number of mitotic cells were described ${ }^{25,41}$. A link between BUBR1 and chromatid separation was also reported in patients with mosaic variegated aneuploidy, a rare disease associated with intrauterine growth retardation, aneuploidy, microcephaly and hydrocephalus $^{26,42}$, further supporting the Diaph3-BubR1nuclear division axis.

What could be the connection between Diaph3 and BubR1? In vitro studies have shown that Diaph3 stabilizes MTs in a APCEB1-dependent manner ${ }^{15,17,43}$. On the other hand, the APC-EB1 complex controls alignment and segregation of chromosomes ${ }^{28,44}$ through interaction with BubR1 (ref. 27). The APC-EB1 complex is also required for centromeric localization of the CPC effector Aurora $\mathrm{B}^{37}$. CPC 'senses' the tension between sister chromatids at the kinetochore ${ }^{30}$. In case of improper connections, Aurora $B$ phosphorylates kinetochore proteins, which destabilizes spindle MT-kinetochore interaction ${ }^{31,32}$. Hence, Diaph3, APC, EB1, BubR1 and CPC proteins belong to a molecular toolkit that regulates interactions between the kinetochore and spindle MTs, as well as the tightness of the mitotic checkpoint.

In addition to the cortical phenotype, Diaph3 mutant embryos display growth retardation, twisting neural tube, facial deformities and increased number and size of brain blood vessels. More than 97\% of mutants die before E14.5, most probably from a severe anaemia due to loss of erythroid cells. Animals that survive until young adulthood exhibit smaller brain, hydrocephalus and growth retardation. These features are common findings in mouse models of microcephaly ${ }^{45,46}$ and in patients with type II microcephalic osteodysplastic primordial dwarfism ${ }^{47}$, whose life is imperiled by modifications of cerebral blood vessels often resulting in stroke or aneurysm. The severity of the Diaph3 phenotype (that is, embryonic lethality) might have precluded the establishment of a causal link between microcephaly and DIAPH3 loss-of-function mutations. Although mutations in DIAPH1 were associated with microcephaly in humans ${ }^{48,49}$, neither Diaph1 or Diaph2 single ko, nor Diaph1 and Diaph2 dko have symptoms of primary microcephaly ${ }^{8,48}$. The involvement of Diaph3 in microcephaly suggests a functional redundancy and/or divergent roles of Diaphanous genes in humans and mice.

In conclusion, our results provide evidence that Diaph3 protects cortical progenitors against mitotic error-induced apoptosis, by preserving the activity of the spindle checkpoint (see model in Fig. 7). Loss of Diaph3 function does not trigger nuclear division errors in the strict sense. Such events occur physiologically, especially in fast dividing cells such as mammalian cortical progenitors. Rather, the lack of Diaph3 loosens the spindle checkpoint enabling a fraction of aberrantly dividing cells, which normally halt in metaphase until nuclear segregation is properly completed, to 'slip' into anaphase, causing aneuploidy and /or mitotic catastrophe.

\footnotetext{
Methods

Animals. All procedures were carried out in accordance with European guidelines and approved by the animal ethics committee of the Université catholique de Louvain. Mouse lines used in this study were: Diaph 3 ko and Diaph $3^{f / f}$

(Supplementary Fig. 1a), Emx1-Cre (B6.Cg-Emx1 ${ }^{\text {tm1(cre)Krj/J; Jackson Lab) }}{ }^{22}$ and FVB-Tg(CAG-Diaph3)924/Lesp/J (Jackson Lab). For Hypoxyprobe staining, Diaph3 heterozygous timed pregnant females were injected with $60 \mathrm{mg} \mathrm{kg}^{-1}$ pymonidazole hydrochloride and E10.5 embryos were harvested and fixed after $45 \mathrm{~min}$. As a positive control, we injected subcutaneously $1 \times 10^{6} \mathrm{~B} 16 \mathrm{~F} 10$ cells in a $\mathrm{C} 57 \mathrm{BL} / 6 \mathrm{~J}$ mouse and collected the tumour 10 days after injection.
}

In situ hybridization. A PCRII-Topo Diaph3 plasmid (Addgene \#45602) was used to produce a UTP ${ }^{33}$-labelled probe as described previously ${ }^{50,51}$. Coronal and sagittal cryosections from E10.5 and E13.5 embryos, and P0 brains were treated with $1 \mu \mathrm{g} \mathrm{ml}^{-1}$ proteinase $\mathrm{K}$ in $0.1 \mathrm{M}$ Tris $\mathrm{HCl} \mathrm{pH} 8$ and $10 \mathrm{mM}$ EDTA, rinsed in diethyl pyrocarbonate (DEPC)-treated water and acetylated for $10 \mathrm{~min}$ at room temperature in $0.25 \mathrm{M}$ acetic anhydride- $0.1 \mathrm{M}$ triethanolamine. Slides were incubated overnight at $65^{\circ} \mathrm{C}$ in a humid chamber with denatured probes $\left(1 \mu \mathrm{g} \mathrm{ml}^{-1}\right)$ in hybridization solution (50\% formamide, $10 \%$ dextran sulphate, $0.3 \mathrm{M} \mathrm{NaCl}, 20 \mathrm{mM}$ Tris $\mathrm{HCl} \mathrm{pH} 7.5,5 \mathrm{mM}$ EDTA, $1 \times$ Denhardt's solution, $0.6 \mathrm{mg} \mathrm{ml}^{-1}$ yeast transfer RNA and $0.1 \%$ SDS). Slides were washed for $30 \mathrm{~min}$ at $65^{\circ} \mathrm{C}$ in $50 \%$ formamide- $2 \times \mathrm{SSC}$, rinsed in $2 \times \mathrm{SSC}$ and treated for $1 \mathrm{~h}$ at $37^{\circ} \mathrm{C}$ with $1 \mu \mathrm{g} \mathrm{ml}^{-1}$ RNAse in NTE buffer $(0.5 \mathrm{M} \mathrm{NaCl}, 10 \mathrm{mM}$ Tris $\mathrm{HCl} \mathrm{pH} 7.5$ and $5 \mathrm{mM}$ EDTA). Slides were washed in $2 \times \mathrm{SSC}$ and $0.2 \times \mathrm{SSC}$ at $65^{\circ} \mathrm{C}$ for $1 \mathrm{~h}$ each, dehydrated with ethanol and exposed to Kodak Biomax Maximum Resolution films (Sigma).

Immunohistochemistry. For histology, E12.5 and E13.5 were fixed in Bouin's solution and processed for haematoxylin-eosin staining. For immunohistochemistry, embryos were fixed in $4 \%$ paraformaldehyde. Cryosections (16-18 $\mu \mathrm{m}$ thick) were processed for antigen retrieval in $0.01 \mathrm{M}$ sodium citrate $(\mathrm{pH} 6)$ for $5 \mathrm{~min}$, washed with PBS and blocked in PBS supplemented with $0.3 \%$ Triton X-100 and 5\% normal goat serum. Slides were incubated overnight at $4{ }^{\circ} \mathrm{C}$ in the same blocking solution containing primary antibodies, washed and incubated with appropriate Alexa Fluor-conjugated secondary antibodies (Invitrogen). Primary antibodies were as follows: Pax6 (Covance; catalogue number PRB-278 P; 1:100), Tbr2 (Abcam; catalogue number ab23345; 1:500), Tbrl (Abcam; Cat catalogue number ab31940; 1:500), active Caspase-3 (Cell Signaling; catalogue number 9661; 1:500), active Caspase-9 (Cell Signaling; catalogue number 9509; 1:500), Survivin (Cell Signaling; catalogue number 2808; 1:400), phospho-Vimentin (Abcam; catalogue number ab22651; 1:50), Doublecortin (Cell Signaling; catalogue number 4604; 1:400), pHH3 (Cell Signaling; catalogue number 9701; 1:100), Tuj-1 (Covance, catalogue number MMS-435 P-0250; 1:1,000), GFP (AVES; catalogue number GFP-1020; 1:1,000), Aurora-B (Abcam; catalogue number ab2254; 1:500), Hp-1 (Hypoxyprobe, catalogue number HP-100 mg), BubR1 (BD Bioscience; catalogue number 612502; Clone 9, 1:500) and isolectin GS-IB4-Alexa Fluor 568 Conjugate (Invitrogen; catalogue number I21412; 1:500). TUNEL staining was performed using an In Situ Cell Death Detection Kit (Roche, catalogue number 11684795910) according to the manufacturer's instructions. Nuclei were counterstained with DAPI. Images were acquired with an Olympus FV1000 confocal microscope and edited with Photoshop (Adobe Systems). Quantification of stained sections was performed on pictures generated with the same $x, y$ and $z$ size. $Z$-size is set to $3 \mu \mathrm{m}$ in Fig. 1 and $5 \mu \mathrm{m}$ in Fig. 4 .

DNA content analysis. Cortical progenitor cells were collected from E11.5 brains and dissociated to single cells using a Neural Tissue Dissociation kit (MACSMiltenyi Biotec) according to the manufacturer's guidelines. Cells were resuspended in PBS, fixed in ice-cold $70 \%$ ethanol for $30 \mathrm{~min}$ on ice and treated with propidium iodide for $30 \mathrm{~min}$ at $37^{\circ} \mathrm{C}$. Analysis was performed with a FACSCanto Flow Cytometer (BD Biosciences).

Culture of embryonic neural progenitor cells. Cortices were isolated from at E10.5 embryos in glucose enriched L-15 and mechanically dissociated in cold PBS. The cells were spun down and suspended in DMEM-F12 supplemented with GlutaMAX, non-essential amino acids, penicillin-streptomycin, pyruvate, B27 minus Vitamin A, N2 (Life Technologies), $10 \mathrm{ng} \mathrm{ml}^{-1}$ basic fibroblast growth factor and $20 \mathrm{ng} \mathrm{ml}^{-1}$ epidermal growth factor (R\&D System) in low cell-binding 96-well round-bottom plates (Sigma). Neurospheres were dissociated to single cells every 4 days using TrypLE (Gibco). Cells were counted and plated in 12-well Cellstar cell culture plates (Greiner) at a density of 150,000 cells per well. To ascertain the neural identity of neurospheres, cells were plated on $12 \mathrm{~mm}$ poly-D-lysine-coated round coverslips (Corning) in 24-well CellStar cell culture plates (Greiner) at a density of 50,000 cells per well. After 4 days, cells were fixed for $15 \mathrm{~min}$ in $2 \%$ paraformaldehyde in PBS, rinsed in PBS, blocked in $5 \%$ normal goat serum, $0.3 \%$ Triton $\mathrm{X}-100 / \mathrm{PBS}$ for $20 \mathrm{~min}$ and incubated overnight at $4{ }^{\circ} \mathrm{C}$ in blocking buffer containing mouse anti-Nestin (1:1,000; Millipore). Cells counterstained with DAPI. Coverslips were mounted with Mowiol.

Preparation of metaphase spreads. Neurospheres (passage 8) were cultured with $0.05 \mu \mathrm{g}$ colcemid per $\mathrm{ml}$ for $3 \mathrm{~h}$, pelleted by centrifugation for $5 \mathrm{~min}$ at 1,000 r.p.m., treated with hypotonic solution $(0.075 \mathrm{M} \mathrm{KCl}$ in water) and fixed in $3: 1$ methanol:acetic acid. Metaphase chromosome spreads were obtained by releasing a few drops of cell suspension onto sulfuric acid (25\%)-cleaned slides. The slides were mounted with Mowiol medium with $0.25 \mu \mathrm{g} \mathrm{ml}^{-1}$ DAPI and examined with an Axioskop fluorescence microscope (Zeiss, Germany).

In utero electroporation. The pEFmEGFP-mDia2 plasmid (Addgene \#25407), coding for a GFP::Diaph3 fusion protein ${ }^{3}$ was introduced in neural progenitor cells via in utero electroporation. E13.5 embryos were injected intraventricularly with $1 \mu \mathrm{g}$ DNA, electroporated according to standard protocols and collected $24 \mathrm{~h}$ later.

Western blotting. Tissues were homogenized in lysis buffer containing $50 \mathrm{mM}$ Tris $\mathrm{HCl} \mathrm{pH} 7.5,150 \mathrm{mM} \mathrm{NaCl}, 1 \mathrm{mM}$ EDTA, $1 \%$ Triton-X 100 and protease inhibitors (Roche). Cell lysates were centrifuged at $13,000 \mathrm{~g}$ for $15 \mathrm{~min}$ at $4{ }^{\circ} \mathrm{C}$. 
a

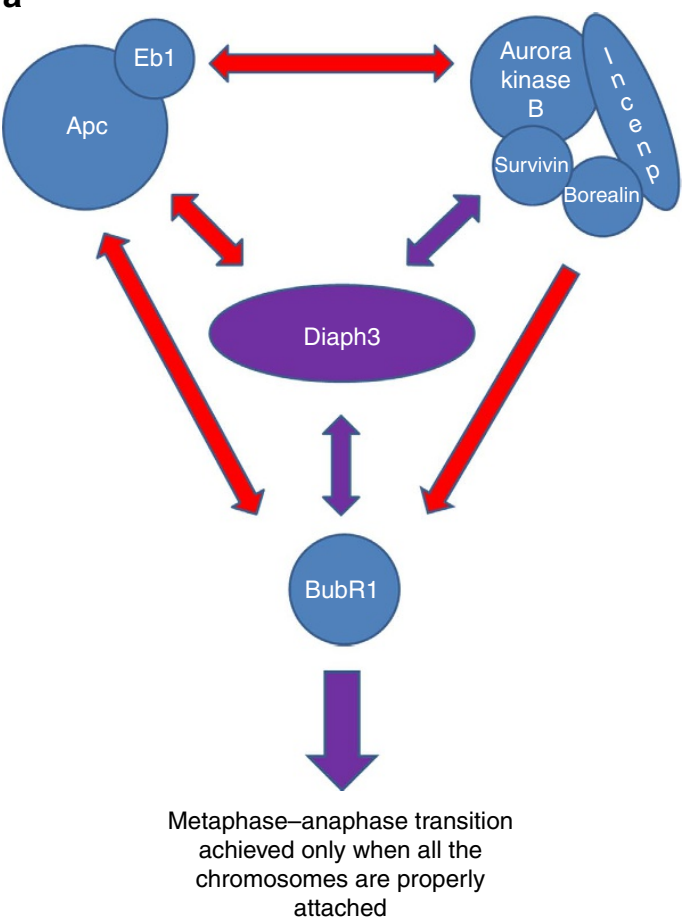

b

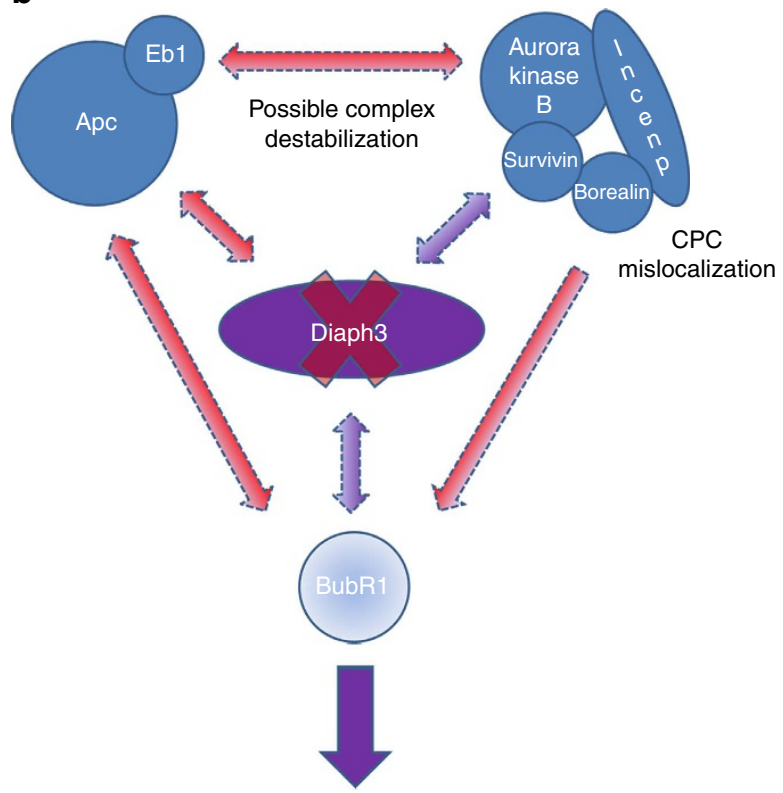

Leaky checkpoint.

Unregulated progression

to anaphase, mitotic

catastrophe or death in

the next G1

Figure 7 | Working model of Diaph3 function in the spindle checkpoint. (a) Molecular interactions between Diaph3, CPC and SAC proteins during cell division. Documented interactions are depicted in red and new findings are shown in purple. Diaph3 localizes with CPC proteins and co-immunoprecipitates with Survivin and BubR1. (b) Absence of Diaph3 probably disrupts the Diaph3-APC-Eb1 complex, which interacts with and stabilizes the CPC complex at the spindle-kinetochore interface. Loss of Diaph3 impairs the localization of CPC proteins and the ability of dividing cells to activate the SAC.

Diaph3-deficient cells fail to accumulate BubR1, leading to slippage to anaphase and mitotic catastrophe and/or cell death of aneuploid progeny.

Protein quantification was performed with a BCA kit (Pierce). Supernatant was mixed with $5 \times$ SDS-loading buffer and heated at $95^{\circ} \mathrm{C}$ for $5 \mathrm{~min}$. Equal amount of proteins were loaded on 10 or $4-20 \%$ TGX gels (Bio-Rad), separated by SDS-PAGE and transferred to nitrocellulose membranes (GE Healthcare). Membranes were blocked with $5 \%$ fat-free dry milk and incubated overnight at $4{ }^{\circ} \mathrm{C}$ with rabbit anti-Diaph3 C-terminal $(1: 5,000)^{3}$, mouse anti-BubR1 (BD Bioscience; catalogue number 612502; Clone 9, 1:500), mouse anti-alpha-tubulin (Sigma; catalogue number T 6199; Clone DM1A; 1:2,000) or chicken anti-GAPDH (Millipore; AB2302; 1:2,000). Relative BubR1 protein quantification was performed using the ImageJ Gel analyser tool. Values were normalized to GAPDH or $\alpha$-tubulin. The amount of BubR1 protein in controls was set to one. Images of western blottings have been cropped for presentation. Full-size images are presented in Supplementary Fig. 11.

Immunoprecipitation. Brain or cortical lysates obtained from $\mathrm{FVB}-\mathrm{Tg}(\mathrm{CAG}-$ Diaph3)924/Lesp/J mice, which constitutively overexpress Diaph3, were prepared as described above. Proteins were quantified using the BCA kit (Pierce). Two hundred micrograms of proteins were incubated overnight in lysis buffer containing protease inhibitors and $5 \mu \mathrm{g}$ rabbit anti-Diaph3 antibody. As negative control for the IP, the lysate was treated in the same conditions but without antibody. Immune complexes from anti-Diaph3 and control IP were recovered by incubation $\left(2 \mathrm{~h}\right.$ at $\left.4{ }^{\circ} \mathrm{C}\right)$ with $20 \mu \mathrm{l}$ protein A-coated agarose beads (Invitrogen) and centrifugation $(2 \mathrm{~min}$ at $2,500 \mathrm{~g})$. Beads were washed three times in PBS and proteins were eluted by addition of $5 \times$ Laemmli buffer and heating at $95^{\circ} \mathrm{C}$ for $5 \mathrm{~min}$. The samples were processed for western blotting, for Survivin and BubR1 proteins. Total brain or cortical lysate were used to immunoprecipitate Survivin and BubR1, respectively.

Lentiviral vector preparation and infection. Lentiviral particles have been prepared as reported ${ }^{52}$. Briefly, HEK293T cells (ATCC CRL-3216) were transiently transfected with Lipofectamine 2000 (Invitrogen) using a third-generation system with lentiviral pHIV transfer vectors together with packaging plasmids pENV, pMDL and pREV. pHIV-IRES-EGFP and pHIV-BubR1-IRES-EGFP transfer plasmids were used to generate lentiviral particles. The first was produced by replacing the dTomato cDNA of a pHIV-dTomato vector (Addgene plasmid \#21374) with an IRES-EGFP cassette from a pIRES2-EGFP plasmid (Clontech). The second was obtained following the same strategy, but after insertion of the mouse BubR1 ORF (Origene) upstream of the IRES-EGFP. Twenty-four and $48 \mathrm{~h}$ after transfection, the virus containing supernatant was harvested, concentrated using $50 \mathrm{kDa}$ Amicon filters (Millipore), titrated and applied to dissociated Diaph3 ko neurospheres for $48 \mathrm{~h}$. Cells were then rinsed several times with PBS and fresh medium was added. Fluorescent neurospheres infected with IRES-EGFP (Control) or BubR1-IRES-EGFP (Rescued) were amplified for four passages, imaged with a Zeiss inverted microscope Axiovert.A1 and processed for karyotype analysis.

BubR1 downregulation in MEFs. MEFs from Diaph $3 \mathrm{TG}$ and WT littermates were isolated following standard protocols. Briefly, E13.5 embryos were eviscerated, minced in small pieces, trypsinized for $30 \mathrm{~min}$ and mechanically dissociated. Cells were cultured in ko-DMEM, supplemented with GlutaMAX, non-essential amino acids, penicillin-streptomycin, pyruvate and $10 \%$ fetal bovine serum (FBS; Invitrogen). MEFs from 3 Diaph 3 TG and 3 controls were pooled together, seeded in 12-well plates at 50-70\% confluency and cultured for $24 \mathrm{~h}$. Cells were then treated for $48 \mathrm{~h}$ with $1 \mu \mathrm{M}$ of either Accell Control siRNA (5'-UGGUUUACAUG UCGACUAA- $3^{\prime}$ ) or Accell mouse BubR1 Smart Pool siRNAs (5'-CCACUAAG CUCGAAUCCUA-3'; $5^{\prime}$-CUACUAGAAUUAAGUGCUU- ${ }^{\prime} ; 5^{\prime}$-CCUAUGACU AUGUAAAUAA-3'; $5^{\prime}$-GCUUUUACUCUGGAGAUGA-3'; Dharmacon) diluted in Accell Delivery Medium. The medium was replaced with complete medium and cells were grown for $24 \mathrm{~h}$, dissociated with TrypLE and seeded again in 12-well plates. After three cycles of knockdown, MEFs were processed for western blotting or karyotype analysis.

DIAPH3 knockdown in 293T cells. HEK293T cells (ATCC CRL-3216) were cultured in DMEM supplemented with GlutaMAX, penicillin-streptomycin and $10 \%$ FBS (Invitrogen), amplified in $25 \mathrm{ml}$ flasks (Greiner) and seeded in 24-well plates (Greiner) at a confluency of $50-70 \%$. Cells were treated for $48 \mathrm{~h}$ with $1 \mu \mathrm{M}$ of either Accell Control siRNA (5'-UGGUUUACAUGUCGACUAA-3') or Accell human DIAPH3 Smart Pool siRNAs (5'-CUACAAGCUUUUAAGUCUC- $3^{\prime}$; 5'-GGAUUUGCUUUGUAAACUU-3'; $5^{\prime}$-GCGUUUAUUAGAAAUGAAG-3'; $5^{\prime}$-CUGAUAUACUGAAUUUUGU-3'; Dharmacon), diluted in Pro293a-CD medium (Lonza) supplemented with GlutaMAX and $2 \%$ FBS. Medium was replaced with complete medium and cells were grown for $24 \mathrm{~h}$, dissociated with TrypLE and seeded again in 12-well plates. Effective DIAPH3 knockdown was checked twice at passage 2 and 3 via western blot analysis. After four cycles of knockdown (16 days of continuous DIAPH3 downregulation), HEK cells were processed for karyotype analysis. 
Statistics. For analysis of different conditions or genotypes, the Student's $t$-test was used. For CPC mislocalization, BubR1 relative amount, postmetaphasic fraction of mitotic NE cells and flow cytometry data, the Fisher's exact test and $z$-test were used. Plotted data are represented as mean \pm s.e.m. Significance levels are as follows: ${ }^{\star} P<0.05,{ }^{* *} P<0.01$ and ${ }^{* *} P<0.001$.

Data availability. Data sharing not applicable to this article. All data were included in the main manuscript and Supplementary Information, and no data sets were generated during the current study.

\section{References}

1. Bogdan, S., Schultz, J. \& Grosshans, J. Formin' cellular structures: physiological roles of diaphanous (Dia) in actin dynamics. Commun. Integr. Biol. 6, e27634 (2013).

2. Sagot, I., Klee, S. K. \& Pellman, D. Yeast formins regulate cell polarity by controlling the assembly of actin cables. Nat. Cell Biol. 4, 42-50 (2002).

3. Tominaga, T. et al. Diaphanous-related formins bridge rho GTPase and Src tyrosine kimase signaling. Mol. Cell 5, 13-25 (2000).

4. Watanabe, S. et al. $\mathrm{mDia} 2$ induces the actin scaffold for the contractile ring and stabilizes its position during cytokinesis in NIH 3T3 cells. Mol. Biol. Cell 19, 2328-2338 (2008).

5. Castrillon, D. H. et al. Toward a molecular genetic analysis of spermatogenesis in Drosophila melanogaster: characterization of male-sterile mutants generated by single P element mutagenesis. Genetics 135, 489-505 (1993).

6. Alberts, A. S. Identification of a carboxyl-terminal diaphanous-related formin homology protein autoregulatory domain. J. Biol. Chem. 276, 2824-2830 (2001)

7. Castrillon, D. H. \& Wasserman, S. A. Diaphanous is required for cytokinesis in Drosophila and shares domains of similarity with the products of the limb deformity gene. Development 120, 3367-3377 (1994).

8. Shinohara, R. et al. A role for $\mathrm{mDia}$, a Rho-regulated actin nucleator, in tangential migration of interneuron precursors. Nat. Neurosci. 15, 373-380 S1-S2 (2012).

9. Watanabe, S. et al. Rho and anillin-dependent control of mDia2 localization and function in cytokinesis. Mol. Biol. Cell 21, 3193-3204 (2010).

10. Watanabe, S. et al. Loss of a Rho-regulated actin nucleator, mDia2, impairs cytokinesis during mouse fetal erythropoiesis. Cell Rep. 5, 926-932 (2013).

11. Schoen, C. J., Burmeister, M. \& Lesperance, M. M. Diaphanous homolog 3 (Diap3) overexpression causes progressive hearing loss and inner hair cell defects in a transgenic mouse model of human deafness. PLOS ONE 8, e56520 (2013)

12. Schoen, C. J. et al. Increased activity of Diaphanous homolog 3 (DIAPH3)/ diaphanous causes hearing defects in humans with auditory neuropathy and in Drosophila. Proc. Natl Acad. Sci. USA 107, 13396-13401 (2010).

13. Vorstman, J. A. et al. A double hit implicates DIAPH3 as an autism risk gene. Mol. Psychiatry 16, 442-451 (2011).

14. Palazzo, A. F., Cook, T. A., Alberts, A. S. \& Gundersen, G. G. mDia mediates Rho-regulated formation and orientation of stable microtubules. Nat. Cell Biol. 3, 723-729 (2001)

15. Bartolini, F. et al. The formin mDia2 stabilizes microtubules independently of its actin nucleation activity. J. Cell Biol. 181, 523-536 (2008).

16. Bartolini, F., Ramalingam, N. \& Gundersen, G. G. Actin-capping protein promotes microtubule stability by antagonizing the actin activity of mDial. Mol. Biol. Cell 23, 4032-4040 (2012).

17. Wen, Y. et al. EB1 and APC bind to mDia to stabilize microtubules downstream of Rho and promote cell migration. Nat. Cell Biol. 6, 820-830 (2004).

18. Florio, M. \& Huttner, W. B. Neural progenitors, neurogenesis and the evolution of the neocortex. Development 141, 2182-2194 (2014).

19. Spector, M. S., Desnoyers, S., Hoeppner, D. J. \& Hengartner, M. O. Interaction between the C. elegans cell-death regulators CED-9 and CED-4. Nature 385, 653-656 (1997)

20. Chu, Z. L. et al. A novel enhancer of the Apaf1 apoptosome involved in cytochrome c-dependent caspase activation and apoptosis. J. Biol. Chem. 276, 9239-9245 (2001).

21. Rodriguez, J. \& Lazebnik, Y. Caspase-9 and APAF-1 form an active holoenzyme. Genes Dev. 13, 3179-3184 (1999).

22. Gorski, J. A. et al. Cortical excitatory neurons and glia, but not GABAergic neurons, are produced in the Emx1-expressing lineage. J. Neurosci. 22, 6309-6314 (2002)

23. Vitale, I., Galluzzi, L., Castedo, M. \& Kroemer, G. Mitotic catastrophe: a mechanism for avoiding genomic instability. Nat. Rev. Mol. Cell Biol. 12, 385-392 (2011)

24. Khan, M. A. et al. A missense mutation in the PISA domain of HsSAS-6 causes autosomal recessive primary microcephaly in a large consanguineous Pakistani family. Hum. Mol. Genet. 23, 5940-5949 (2014).

25. Dai, W. et al. Slippage of mitotic arrest and enhanced tumor development in mice with BubR1 haploinsufficiency. Cancer Res. 64, 440-445 (2004).
26. Matsuura, S. et al. Monoallelic BUB1B mutations and defective mitotic-spindle checkpoint in seven families with premature chromatid separation (PCS) syndrome. Am. J. Med. Genet. A 140A, 358-367 (2006).

27. Zhang, J., Ahmad, S. \& Mao, Y. BubR1 and APC/EB1 cooperate to maintain metaphase chromosome alignment. J. Cell Biol. 178, 773-784 (2007).

28. Draviam, V. M., Shapiro, I., Aldridge, B. \& Sorger, P. K. Misorientation and reduced stretching of aligned sister kinetochores promote chromosome missegregation in EB1- or APC-depleted cells. EMBO J. 25, 2814-2827 (2006).

29. Stepanenko, A. A. \& Dmitrenko, V. V. HEK293 in cell biology and cancer research: phenotype, karyotype, tumorigenicity, and stress-induced genome-phenotype evolution. Gene 569, 182-190 (2015).

30. Lampson, M. A. \& Cheeseman, I. M. Sensing centromere tension: Aurora B and the regulation of kinetochore function. Trends Cell Biol. 21, 133-140 (2011).

31. Wang, E., Ballister, E. R. \& Lampson, M. A. Aurora B dynamics at centromeres create a diffusion-based phosphorylation gradient. J. Cell Biol. 194, 539-549 (2011).

32. Afonso, O. et al. Feedback control of chromosome separation by a midzone Aurora B gradient. Science 345, 332-336 (2014).

33. Shao, X., Kawauchi, K., Shivashankar, G. V. \& Bershadsky, A. D. Novel localization of formin mDia2: importin beta-mediated delivery to and retention at the cytoplasmic side of the nuclear envelope. Biol. Open 4, 1569-1575 (2015).

34. DeWard, A. D. \& Alberts, A. S. Ubiquitin-mediated degradation of the formin mDia2 upon completion of cell division. J. Biol. Chem. 284, 20061-20069 (2009).

35. Liu, C. \& Mao, Y. Diaphanous formin mDia2 regulates CENP-A levels at centromeres. J. Cell Biol. 213, 415-424 (2016).

36. Banerjee, B., Kestner, C. A. \& Stukenberg, P. T. EB1 enables spindle microtubules to regulate centromeric recruitment of Aurora B. J. Cell Biol. 204, 947-963 (2014).

37. Sun, L. et al. EB1 promotes Aurora-B kinase activity through blocking its inactivation by protein phosphatase 2A. Proc. Natl Acad. Sci. USA 105, 7153-7158 (2007).

38. Gruneberg, U., Neef, R., Honda, R., Nigg, E. A. \& Barr, F. A. Relocation of Aurora B from centromeres to the central spindle at the metaphase to anaphase transition requires MKlp2. J. Cell Biol. 166, 167-172 (2004).

39. Hager, M. H. et al. DIAPH3 governs the cellular transition to the amoeboid tumour phenotype. EMBO Mol. Med. 4, 743-760 (2012).

40. Morley, S. et al. Regulation of microtubule dynamics by DIAPH3 influences amoeboid tumor cell mechanics and sensitivity to taxanes. Sci. Rep. 5, 12136 (2015).

41. Baker, D. J. et al. BubR1 insufficiency causes early onset of aging-associated phenotypes and infertility in mice. Nat. Genet. 36, 744-749 (2004).

42. Miyamoto, T. et al. Insufficiency of BUBR1, a mitotic spindle checkpoint regulator, causes impaired ciliogenesis in vertebrates. Hum. Mol. Genet. 20, 2058-2070 (2011).

43. Eng, C. H., Huckaba, T. M. \& Gundersen, G. G. The formin mDia regulates GSK3beta through novel PKCs to promote microtubule stabilization but not MTOC reorientation in migrating fibroblasts. Mol. Biol. Cell 17, 5004-5016 (2006).

44. Green, R. A., Wollman, R. \& Kaplan, K. B. APC and EB1 function together in mitosis to regulate spindle dynamics and chromosome alignment. Mol. Biol. Cell 16, 4609-4622 (2005).

45. Murga, M. et al. A mouse model of ATR-Seckel shows embryonic replicative stress and accelerated aging. Nat. Genet. 41, 891-898 (2009).

46. Chen, C. T. et al. A unique set of centrosome proteins requires pericentrin for spindle-pole localization and spindle orientation. Curr. Biol. 24, 2327-2334 (2014).

47. Hall, J. G., Flora, C., Scott, Jr C. I., Pauli, R. M. \& Tanaka, K. I. Majewski osteodysplastic primordial dwarfism type II (MOPD II): natural history and clinical findings. Am. J. Med. Genet. A 130A, 55-72 (2004).

48. Ercan-Sencicek, A. G. et al. Homozygous loss of DIAPH1 is a novel cause of microcephaly in humans. Eur. J. Hum. Genet. 23, 165-172 (2015).

49. Al-Maawali, A. et al. Novel loss-of-function variants in DIAPH1 associated with syndromic microcephaly, blindness, and early onset seizures. Am. J. Med Genet. A 170A, 435-440 (2016).

50. Chai, G. et al. Celsr3 is required in motor neurons to steer their axons in the hindlimb. Nat. Neurosci. 17, 1171-1179 (2014).

51. Zhou, L. et al. Early forebrain wiring: genetic dissection using conditional Celsr3 mutant mice. Science 320, 946-949 (2008)

52. Taulli, R. et al. RNAi technology and lentiviral delivery as a powerful tool to suppress Tpr-Met-mediated tumorigenesis. Cancer Gene Ther. 12, 456-463 (2005).

\section{Acknowledgements}

We thank Paolo Porporato for xenografts and help with production of lentiviral particles Yves Jossin and members of the Developmental Neurobiology group for discussions; and Valérie Bonte, Rachid El Kaddouri, Isabelle Lambermont and Esther Paitre for technical support. This work was supported by the following grants: FNRS PDR T0002.13, FNRS 
PDR T00075.15, Interuniversity Poles of Attraction (SSTC, PAI p6/20 and PAI7/20), Fondation Médicale Reine Elisabeth, Fondation JED-Belgique and WELBIO-CR-

2012A-07, all from Belgium. F.T. is a senior research associate of the Belgian Funds for Scientific Research (FRS-FNRS).

\section{Author contributions}

D.D. performed most of experiments. F.T. produced Diaph 3 ko mice and carried out in situ hybridization and chromosome content analysis. D.D., A.M.G. and F.T. designed research. A.A. provided fundamental reagents. D.D. and F.T. wrote the manuscript. All authors edited and approved the manuscript.

\section{Additional information}

Supplementary Information accompanies this paper at http://www.nature.com/ naturecommunications

Competing financial interests: The authors declare no competing financial interests.
Reprints and permission information is available online at http://npg.nature.com/ reprintsandpermissions/

How to cite this article: Damiani, D. et al. Lack of Diaph3 relaxes the spindle checkpoint causing the loss of neural progenitors. Nat. Commun. 7, 13509 doi: 10.1038/ncomms13509 (2016).

Publisher's note: Springer Nature remains neutral with regard to jurisdictional claims in published maps and institutional affiliations.

(c) (i) This work is licensed under a Creative Commons Attribution 4.0 International License. The images or other third party material in this article are included in the article's Creative Commons license, unless indicated otherwise in the credit line; if the material is not included under the Creative Commons license, users will need to obtain permission from the license holder to reproduce the material. To view a copy of this license, visit http://creativecommons.org/licenses/by/4.0/

(C) The Author(s) 2016 